\title{
The Prevalence of NAFLD and Fibrosis in Bariatric Surgery Patients and the Reliability of Noninvasive Diagnostic Methods
}

\author{
Maurizio Soresi, ${ }^{1}$ Daniela Cabibi ${ }^{D},{ }^{1}$ Rosaria V. Giglio, ${ }^{1}$ Stefania Martorana, ${ }^{2}$ \\ Giuseppina Guercio, ${ }^{2}$ Rossana Porcasi, ${ }^{1}$ Antonino Terranova, ${ }^{1}$ Luigi A. Lazzaro, ${ }^{2}$ \\ Maria R. Emma, ${ }^{3}$ Giuseppa Augello, ${ }^{3}$ Melchiorre Cervello $\mathbb{D}^{3}$ Gianni Pantuso, ${ }^{2}$ \\ Giuseppe Montalto, ${ }^{1,3}$ and Lydia Giannitrapani ${ }^{1}{ }^{1,3}$ \\ ${ }^{1}$ Department of Health Promotion Sciences, Maternal and Infant Care, Internal Medicine and Medical Specialties (PROMISE), \\ University of Palermo, Palermo, Italy \\ ${ }^{2}$ Department of Surgical Oncological and Oral Sciences, Division of General and Oncological Surgery, University of Palermo, \\ Palermo, Italy \\ ${ }^{3}$ Institute for Biomedical Research and Innovation (IRIB), National Research Council, Palermo, Italy
}

Correspondence should be addressed to Lydia Giannitrapani; lydiagiannitp@gmail.com

Received 16 January 2020; Revised 19 March 2020; Accepted 27 March 2020; Published 27 April 2020

Academic Editor: Stefano Gitto

Copyright $\odot 2020$ Maurizio Soresi et al. This is an open access article distributed under the Creative Commons Attribution License, which permits unrestricted use, distribution, and reproduction in any medium, provided the original work is properly cited.

\begin{abstract}
Background. Bariatric surgery patients have a higher prevalence of nonalcoholic fatty liver (NAFL) than the general population; however, its assessment and the accurate staging of fibrosis are often complicated because noninvasive tests are not very accurate in patients with morbid obesity, and liver biopsy cannot be performed as a routine exam. The aim of this study was to evaluate (A) the histological prevalence of NAFL, nonalcoholic steatohepatitis (NASH), and fibrosis in patients undergoing bariatric surgery; (B) the reliability of ultrasound (US) in diagnosing NAFL; and (C) the reliability of various fibrosis scoring systems for defining fibrosis. Methods. US and intraoperative liver biopsy results were reviewed in 57 bariatric surgery patients. NAFL, NASH, and fibrosis were diagnosed according to the Kleiner scoring system. US diagnosis of liver steatosis was based on the bright liver. Fibrosis scores used were (i) the BMI, AST/ALT Ratio, Diabetes (BARD) scoring system; (ii) the nonalcoholic fatty liver disease (NAFLD) fibrosis score; and (iii) the fibrosis-4 (FIB-4) index. Results. The prevalence of NAFL was $81 \%$, NASH $61.4 \%$, and fibrosis $94 \%$ (F3 5.7\%, cirrhosis $2.8 \%$ ). The sensitivity of US was $95 \%$, specificity $50 \%$, and likelihood ratio (LR+, LR-) 1.91 and 0.1 . The reliability of fibrosis scores for $F \geq 2$ were as follows: BARD score: sensitivity $46 \%$, specificity $54 \%$, and area under the receiver-operating characteristics (AUROC) curve 0.5; NAFLD score: sensitivity 30\%, specificity $89 \%$, and AUROC 0.5; and FIB-4: sensitivity 68\%, specificity 67\%, and AUROC 0.7. Conclusions. In bariatric surgery patients, the prevalence of NAFL was $81 \%$, NASH $61.4 \%$, and fibrosis $94 \%$. US is able to rule out the presence of NAFL, while the commonly used scores may be inaccurate in defining fibrosis in patients with morbid obesity.
\end{abstract}

\section{Introduction}

Nonalcoholic fatty liver disease (NAFLD) is a very common chronic liver disease worldwide, and in Western countries, it is the most frequent type of liver disease [1-7]. Diagnosis is based on an abnormal accumulation of fat in more than $5 \%$ of hepatocytes at liver biopsy, in the absence of other causes such as alcohol abuse, viral infection, autoimmune, or drug-related liver diseases. Since being overweight, obesity (especially visceral), dyslipidemia, and diabetes mellitus are recognized as risk factors for NAFLD, it is considered a component of metabolic syndrome (MS) [7-9].

The term NAFLD covers a wide spectrum of diseases, ranging from simple steatosis (NAFL) to nonalcoholic steatohepatitis (NASH), in which steatosis is associated with ballooning degeneration of hepatocytes and diffuse 
lobular inflammation; this is the form that can evolve towards liver fibrosis of varying degrees of severity, up to cirrhosis and hepatocellular carcinoma (HCC) [1-6].

Morbid obesity is often defined as a body mass index (BMI) exceeding $35 \mathrm{~kg} / \mathrm{m}^{2}$ combined with at least one obesity-associated disease, or a BMI exceeding $40 \mathrm{~kg} / \mathrm{m}^{2}$ with or without obesity-associated diseases [10]. NAFLD occurs frequently in people with morbid obesity, and it has been reported in $80-90 \%$ of patients undergoing bariatric surgery. An estimated $20-47 \%$ of these patients have NASH, of which $8-12 \%$ progress to cirrhosis and an undefined percentage to HCC [8-10]. From these data, it is clear that NAFLD, in its various stages of evolution, can adversely affect the health and survival of these patients. Therefore, the accurate evaluation of the presence of steatosis and the correct staging of fibrosis are important for the accurate assessment of these subjects' prognoses.

The gold standard for diagnosing steatosis and fibrosis is liver biopsy, but this is not always feasible as, in most patients, liver disease is asymptomatic and often without laboratory indications [1]. Several noninvasive tests have been proposed for defining fibrosis [1], and for the diagnosis of fatty liver disease, ultrasound (US) is certainly a reliable method, well accepted by patients and sufficiently accurate [10-14]. However, for fibrosis, elastography, considered the most reliable method for diagnosing chronic liver disease, is not sufficiently accurate in people with morbid obesity. Consequently, a number of alternative scores have been proposed [1, 15]. The most commonly used of these are the BMI, AST/ALT Ratio, Diabetes (BARD) scoring system, the NAFLD fibrosis score, and the fibrosis-4 (FIB-4) index [1, 16-18].

The aim of our study was to retrospectively evaluate (A) the histological prevalence of liver steatosis and fibrosis in a population of patients undergoing bariatric surgery; (B) the reliability of US in the diagnosis of steatosis; (C) the reliability of the various fibrosis scores listed above for defining liver fibrosis.

\section{Patients and Methods}

2.1. Patient Selection. We retrospectively examined US liver images and the results of intraoperative liver biopsies in 57 patients undergoing bariatric surgery, consecutively enrolled between January 2015 and December 2018 at the Bariatric Surgery Unit of the University Hospital of Palermo, Italy. The study was conducted in accordance with the ethical standards laid down in the Helsinki Declaration of 1975 and its subsequent amendments and was approved by the Ethics Committee as a spontaneous study on 23 June 2014 (No. 7/2014). All patients gave their approval and signed informed consent.

Before surgery, all patients had been interviewed and examined during an outpatient visit. A standard questionnaire was used to investigate the presence of metabolic, cardiovascular, or liver diseases, use of any hepatotoxic drugs, and alcohol consumption.

The patients then underwent a physical examination in which weight $(\mathrm{kg})$, height $(\mathrm{m})$, and BMI were measured.
Blood pressure was evaluated with the patient in a sitting position, and hypertension was diagnosed according to the WHO/ISH criteria [19]. A blood sample was also taken from patients the morning before surgery to measure levels of glucose, total cholesterol and HDL, triglycerides, aminotransferases (AST/ALT), and insulin.

Diagnosis of type-2 diabetes mellitus (T2DM), impaired fasting glycemia (IFG), and impaired glucose tolerance (IGT) was performed in accordance with the expert committee on criteria for the diagnosis and classification of diabetes mellitus [20]. Insulin resistance was calculated using total insulin and glucose levels, following the homeostasis model assessment (HOMA-R) [21]. Patients with elevated transaminase values were tested for anti-HCV, HBsAg, ANA, AMA, ASMA, and LKM1 antibodies.

US was performed by a single operator the morning before surgery, after 10 hours of fasting, using a Philips 5000 HDI US machine with a $2-5 \mathrm{MHz}$ convex multifrequency probe. NAFLD diagnosis was based on the presence of the bright liver on US [7]. Patients were divided into NAFLD-US positives or negatives.

Serum fibrosis scores were obtained using the following methods:

(1) BARD scoring system, which consists of three variables: $\mathrm{BMI}>28,1$ point; AST/ALT ratio $>0.8,2$ points; and T2DM, 1 point. The score varies from 0 to 4 points. According to Harrison et al. [17], scores of 2-4 points are associated with F3 or F4 stages of fibrosis, with an odds ratio of $17.333(95 \% \mathrm{Cl}$; $3.639-82.558$ ) and a negative predictive value of $97 \%$

(2) NAFLD fibrosis score, established with the following formula: $-1.675+0.037-$ age $($ years $)+0.094-$ $\mathrm{BMI}\left(\mathrm{kg} / \mathrm{m}^{2}\right)+1.13 \times \mathrm{IFG} /$ diabetes $($ yes $=1$, no $=0)+$ $0.99 \times$ AST/ALT ratio $-0.013 \times$ platelet count $\left(\times 10^{9} / \mathrm{L}\right)$ $-0.66 \times$ albumin $(\mathrm{g} / \mathrm{dL})$. According to Angulo et al. [18], a score below - 1.455 (low cut-off) excludes advanced fibrosis, while a score higher than 0.676 predicts advanced fibrosis. The scores between these values are defined as indeterminate

(3) FIB-4 index: age $\times \operatorname{AST}(\mathrm{U} / \mathrm{L}) /$ platelets $\left(10^{9} / \mathrm{L} /\right.$ $\sqrt{\operatorname{ALT}(\mathrm{U} / \mathrm{L})}[16]$.

2.2. Liver Histology. Wedge or core liver biopsies to the depth of Glisson's capsule were obtained shortly after the abdominal cavity was opened and before manipulation of the liver.

Liver biopsies were routinely formalin-fixed and paraffinembedded. Hematoxylin and eosin (H\&E) and picrosirius staining were available for each case. Slides were read by one expert pathologist (D.C.) who was unaware of patient identities and histories. A minimum biopsy specimen length of $15 \mathrm{~mm}$ or the presence of at least 10 complete portal tracts was required.

The Kleiner scoring system was used as a protocol for the histological assessment of steatosis [22]. This system evaluates the degree of NAFLD by assigning separate scores for steatosis, hepatocellular ballooning, lobular inflammation, 
and stage of fibrosis. Fibrosis was assessed on the 5-point scale (0-4) suggested by Kleiner et al. [22], in which stages $\mathrm{F} 0=$ absence of fibrosis, $\mathrm{F} 1=$ perisinusoidal or periportal fibrosis, F2 = perisinusoidal and portal/periportal fibrosis, F3 = bridged fibrosis, and F4 = cirrhosis.

The presence of NASH was assessed using the steatosis, activity, and fibrosis (SAF) score [23].

All patients who had an etiologically well-defined liver disease were excluded from the study.

2.3. Statistical Analysis. Data were expressed as mean \pm standard deviation (SD) or as median and range (min-max). The prevalence of steatosis and fibrosis, sensitivity (Se), specificity (Sp), positive predictive value (PPV), negative predictive value (NPV), accuracy (Acc), and positive and negative likelihood ratio ( $\mathrm{LR}+, \mathrm{LR}-)$ were calculated using classical formulas. The receiver-operating characteristic (ROC) curve was constructed by calculating the sensitivity and specificity of individual serum fibrosis tests at different cut-off points, and the corresponding area under the curve (AUROC) was calculated to evaluate the diagnostic accuracy of the tests in differentiating $\mathrm{F} \geq 2$ [24]. The best cut-off value was calculated as the maximum LR obtained using the following formula: $L R=$ probability of true positive + probability of true negative/probability of false positive + probability of false negative [25].

\section{Results}

Table 1 shows the clinical and demographic characteristics of the enrolled patients. Liver steatosis, defined as the accumulation of fatty acid in more than $5 \%$ of hepatocytes, was present in $46 / 57$ biopsies (Figure 1(a)), with a prevalence of $81 \%$ (95\% CI: 71-90). Figure 1(a) shows the steatosis grading according to the Kleiner scoring system. In particular, $<5 \%$ (nonsteatosis, Grade 0) was found in $17.9 \%$ of biopsies; $5-33 \%$ (Grade I) in $30.4 \%$; $33 \%-66 \%$ (Grade II) in 26.8\%; and $>66 \%$ (Grade III) in $25 \%$.

Table 2 shows the diagnostic reliability data of US for steatosis diagnosis. Sensitivity was 95\% (95\% CI: 81-98), specificity 50\% (95\% CI: 18-75), PPV 89.4 (95\% CI: 82-94), NPV 71\% (95\% CI: 36-92), accuracy 87\% (95\% CI: 75-94), positive LR 1.91 (95\% CI: 1.1-3.5), and negative LR 0.1 (95\% CI: 0.02-0.4).

Figure 1(b) shows the prevalence of NASH. NASH was present in $35 / 57$ patients $(61.4 \%$; $95 \% \mathrm{CI}: 58-73 \%)$. Of the 46 patients with steatosis, fibrosis was present in 40 (87\%; 95\% CI: 75-95). In patients with NASH, fibrosis was present in 33/35 (94\%; 95\% CI: 0.89-0.98) (Figure 1(c)), divided as follows: F0 fibrosis in 2 cases (5.7\%; 95\% CI: 1-30\%), F1 in 12 cases (34.1\%; 95\% CI: $21-50 \%)$, F2 in 18 patients (51\%; 95\% CI: $18-35 \%)$, F3 (bridging fibrosis) in 2 patients $(5.7 \%$; 95\% CI: $1-30 \%$ ), and F4 (cirrhosis) in 1 case $(2.8 \% ; 95 \%$ CI: $0.5-20)$.

The AUROC curves of the individual fibrosis tests and the best diagnostic cut-off values were calculated. The reliability of serum fibrosis scores in defining $\mathrm{F} \geq 2$ at the best cut-off values is shown in Table 3. Figure 2 shows the
TABLE 1: Clinical and demographic characteristics of the enrolled patients.

\begin{tabular}{lc}
\hline & $n=57$ \\
\hline Age (y) & $42 \pm 12.0$ \\
M/F & $16 / 41$ \\
BMI & $43.5 \pm 7.1$ \\
Diabetes & $13(14.3 \%)$ \\
Hypertension & $17(29.8 \%)$ \\
HOMA & $4(2.4-6.9)$ \\
AST (U/L) & $18.5(16-27)$ \\
Elevated AST (>40 U/L) & $3(5.26 \%)$ \\
ALT (U/L) & $29(20-39)$ \\
Elevated ALT (>40 U/L) & $9(15.8 \%)$ \\
Total cholesterol (mg/dL) & $192 \pm 28.7$ \\
Triglycerides (mg/dL) & $113(68-140)$ \\
HDL (mg/dL) & $54 \pm 14.0$ \\
LDL (mg/dL) & $115.0 \pm 27.2$ \\
\hline
\end{tabular}

AUROC curve of the FIB-4 index with an AUROC of 0.7 and the best cut-off point.

\section{Discussion}

Liver steatosis is considered the most frequent liver disease, especially in Western countries, and its prevalence is growing worldwide: $44 \%$ in the USA, 33\% in Europe, and 25\% in Italy. NAFLD is associated with obesity, dyslipidemia, and diabetes mellitus, and its prevalence varies according to the populations observed and the diagnostic methods used, including histology, US, elastography, or magnetic resonance imaging [1-7]. In patients with MS and dyslipidemia, a 78\% prevalence of liver steatosis detected by US has been reported. In diabetics, the prevalence was between $30 \%$ and $80 \%$, while in obese patients, it increased to between $81 \%$ and $87 \%$ [1-7].

NAFLD is associated with a higher mortality rate for cardiovascular, liver, and cancer diseases than the general population. In liver disease, the correct evaluation of a patient's histological evolution is crucial in defining prognosis [3]. The presence of fibrosis and its evolution towards cirrhosis negatively impact the survival of NAFLD patients.

The prevalence of steatosis in liver biopsies in our series was $81 \%$, similar to the data reported in the literature. In contrast, the reliability of US in diagnosing steatosis in our study differed from values defined for the general population, where sensitivity has been estimated at $74 \%-91 \%$ and specificity at $85 \%-98 \%$, depending on the case series considered [10-14]. In our study, sensitivity was comparable to data reported for the general population, whereas our $50 \%$ specificity was unsatisfactory. Literature data in the past have reported conflicting results for specificity in diagnosis by US [10, 26, 27], and our study confirmed these doubts. However, our data also highlighted a very interesting fact: LR- and LR+ analyses gave values of 0.1 and 1.9 , respectively. In general, values of $L R-\leq 0.1$ in a diagnostic test indicate a great reliability 


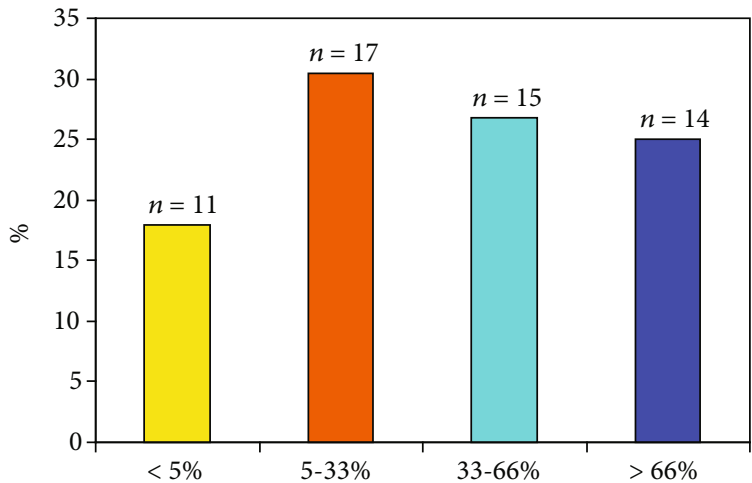

(a)

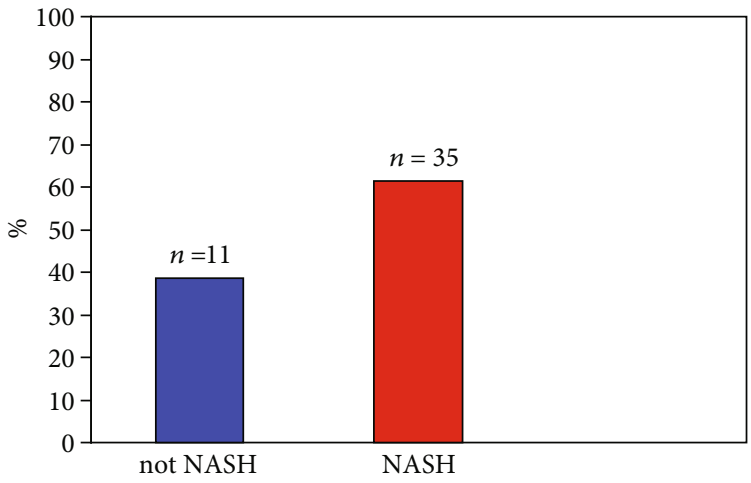

(b)

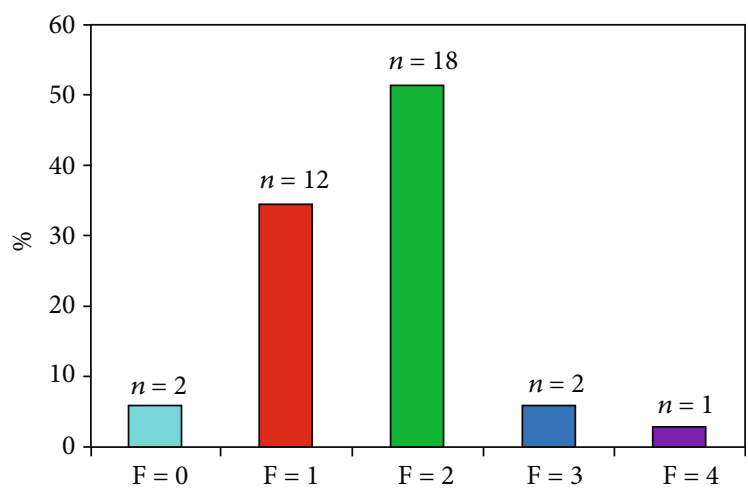

(c)

Figure 1: (a) Steatosis grading according to Kleiner. (b) Diagnosis of NAFLD according to SAF score. (c) Fibrosis score according to Kleiner.

TABLE 2: Diagnostic reliability of ultrasound in the diagnosis of steatosis in the study population.

\begin{tabular}{lc}
\hline & Ultrasound \\
\hline Sensitivity & $95 \%$ (95\% CI: $84-99)$ \\
Specificity & $50 \%$ (95\% CI: $18-81)$ \\
Likelihood ratio + & $1.91 \%$ (95\% CI: $1.1-3.5)$ \\
Likelihood ratio - & $0.1 \%$ (95\% CI: 0.02-0.4) \\
Positive predictive value & $89.4 \%$ (95\% CI: $82-94)$ \\
Negative predictive value & $71 \%$ (95\% CI: $36-92)$ \\
Accuracy & $87 \%$ (95\% CI: $75-94)$ \\
\hline
\end{tabular}

in excluding the disease, whereas values of $L R+>10$ indicate that the test is able to indicate its presence with a high level of confidence. Based on these observations, our finding of $L R-\leq 0.1$ indicates that US has a high reliability in defining the absence of steatosis in obese subjects but that, with a LR+ of 1.9 , it is not very reliable for determining its presence [28, 29].

The limited reliability of US in detecting steatosis in patients with morbid obesity, shown by the low specificity and low LR+, probably depends on a number of factors, mainly related to the physics of US diffusion. It is a known fact that the deeper US waves go, the greater their diffraction and the lower their resolution capacity. In short, the ability of US to define a correct echo pattern is reduced, and very often an incorrect increase in the reg- ulation curve of the gain increases the echogenicity of the liver, producing false positives; the bright liver typical of steatosis is, in fact, hyperechoic.

Studies conducted on obese subjects have reported a 6$94 \%$ frequency of fibrosis and a $26-55 \%$ frequency of NASH [30-34]. In our series, according to the SAF score [23], NASH was present in $61.4 \%$ of patients. Our results are slightly higher than the average in the literature, likely due to various phenomena occurring in patients with morbid obesity, above all insulin resistance because of the greater degree and extent of inflammation. Since there is no reliable serum parameter for $\mathrm{NASH}$, noninvasive parameters to define the presence of steatohepatitis [1] were not evaluated in our series.

The prevalence of fibrosis in all subjects with steatosis was $87 \%$. In the 11 subjects with NAFL alone without inflammation, a fibrosis score of F1 was found. In NASH patients, the prevalence of fibrosis was $94 \%$ (95\% CI: 0.89-0.98), distributed as follows: F1 in 12 cases (34.1\%; 95\% CI: $21-50 \%)$, F2 in 18 patients (51\%; 95\% CI: $18-35 \%)$, F3 (bridging fibrosis) in 2 patients (5.7\%; 95\% CI: $1-30 \%)$, and F4 (cirrhosis) in 1 case $(2.8 \%$; $95 \%$ CI: $0.5-20)$.

These data suggest several considerations. First, it is possible to find small amounts of fibrosis even in patients with NAFL alone, as reported in the literature. This may depend on many factors: the limits of biopsy sampling, or the possibility of finding a disease in phases where the presence of inflammation may be less accentuated. 
TABLE 3: Reliability of serum markers in the definition of moderate fibrosis $(F \geq 2)$.

\begin{tabular}{lccc}
\hline & $\begin{array}{c}\text { NAFLD score } \\
\text { Cut - off }<-1.455 \\
\%(95 \% \text { CI })\end{array}$ & $\begin{array}{c}\text { BARD score } \\
\text { Cut - off } \geq 2 \\
\%(95 \% \text { CI) }\end{array}$ & $\begin{array}{c}\text { Cut }- \text { off }>0.41 \\
\%(95 \% \text { CI })\end{array}$ \\
\hline Sensitivity & $30(12-54)$ & $46(27-67)$ & $68(46-85)$ \\
Specificity & $89(52-99)$ & $54(27-79)$ & $67(52-98)$ \\
Likelihood ratio + & $2.7(1.3-5.5)$ & $0.6(0.1-3)$ & $2(1.3-3.2)$ \\
Likelihood ratio - & $0.8(0.1-5.1)$ & $1.1(0.84-1.34)$ & $0.5(0.1-4.4)$ \\
Positive predictive value & $93(82-97)$ & $50(13-86)$ & $77(45-92)$ \\
Negative predictive value & $22(12-38)$ & $36(31-42)$ & $55(29-78)$ \\
Accuracy & $64.3(48-78.5)$ & $37(23-54)$ & $60(42-74)$ \\
AUROC* & 0.53 & 0.5 & 0.7 \\
\hline
\end{tabular}

${ }^{*}$ The area under the receiver-operating characteristic.

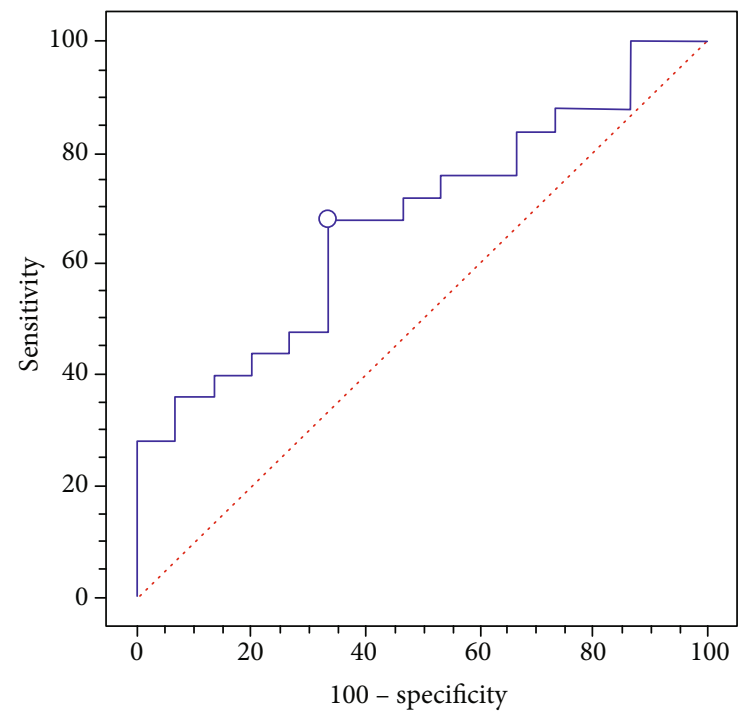

Figure 2: FIB-4 ROC curve with area under the curve of 0.7 and the best cut-off point.

Second, despite the prevalence of fibrosis falling within the range reported by the literature, our percentage of advanced fibrosis $(\geq \mathrm{F} 3)$ was very low.

Although the validity of our considerations is limited by the small study population, this observation may also be due to the relatively young age of our patients (41 years). Age is indeed known to be one of the most important variables influencing NASH onset and evolution; in fact, as age increases, there is also an increased incidence of insulin resistance, MS, and diabetes mellitus [35, 36]. In a systematic review of this issue, Argo et al. found that age was the independent predictor of advanced fibrosis (HR 0.98, 95\% CI: $0.96-0.99, p=0.009$ ) and that young people have a lower risk for the presence of fibrosis [37]. However, this study did not show any relationship between fibrosis and diabetes, in contrast to McPherson et al. who found an important role for diabetes among patients with NAFL: in fact, $80 \%$ of those having fibrosis progression were diabetic at the follow-up liver biopsy compared to $25 \%$ of nonprogressors $(p=0.005)$ [38].
Moreover, there is another reason which may explain the lower frequency of fibrosis in our study: the high prevalence of premenopausal women. Gender and reproductive states may influence the degree of fibrosis in patients with NASH. Longitudinal studies suggest that the incidence of NAFLD is higher in males than in females, and the incidence is higher in menopausal $(7.5 \%)$ /postmenopausal $(6.1 \%)$ women as compared to premenopausal (3.5\%) women [39]. In addition, an Italian multicenter study found that men with NAFLD were approximately 10 years younger than women, confirming previous findings that premenopausal women are somehow "protected" from NAFLD [40].

Yang et al. found an adjusted cumulative odds ratio (ACOR) and 95\% confidence interval (CI) for greater fibrosis severity of $1.4(0.9,2.1)(p=0.17)$ for postmenopausal women, with premenopausal women as a reference [41].

Finally, Wang Z. et al., studying the predictive factors of $\mathrm{NASH}$ and fibrosis in women with different BMI and age, reported that the prevalence of NASH seems to be considerably higher in obese and postmenopausal women with NAFLD [42].

Further, the use of serum markers to evaluate the presence of fibrosis did not yield reliable results. The AUROC values of the BARD and NAFLD scores showed low accuracy. Only the FIB-4 index presented an acceptable ROC curve of 0.7 , but the modest results obtained in our study were not enough to indicate it as being reliable. Our results are in disagreement with the literature, which recognizes an important role for the FIB-4 index and NAFLD score, but it is possible that the young age of our population played an important role in this result, given that the individual's age is included in the calculation of the scores. However, even in this case, some considerations must be made: (1) our limited number of cases may have reduced the reliability of the results, as shown by the wide confidence interval; (2) most of the patients reported in the guidelines did not suffer from morbid obesity [1]; (3) our patients showed a low degree of fibrosis; in fact, the accuracy of noninvasive fibrosis tests is known to be very limited for $\mathrm{F} \leq 2$ values [1]; (4) it possible that the young age of our patients also conditioned the results of the noninvasive fibrosis tests because most of the tools/calculators assessed take the age of the individual into account. 
Finally, we have to underline that our patients were all Italian, and therefore, our results cannot be generalized; in fact, both NASH and its evolution may vary according to ethnicity. For example, Hispanics with obesity and diabetes have a far higher risk for advanced liver disease than other racial or ethnic groups, indicating the potential involvement of other factors such as genetic variants [43].

\section{Conclusions}

In conclusion, our data show that US is a method that can exclude the presence of steatosis in subjects with severe obesity with a good degree of reliability.

Steatosis assessment with the controlled attenuation parameter using XL probes has recently been proposed in obese subjects and seems to have a good level of accuracy. It is advisable, in our opinion, to reserve this examination for patients in whom US images suggest steatosis, in order to confirm and eventually stage it.

The literature data report that, even in subjects with obesity, fibrosis serum markers are reliable; however, in our study, the accuracy of fibrosis evaluation with serum markers appeared to be limited, though this may be due to the low prevalence of fibrosis in our patients. In any case, the noninvasive evaluation of fibrosis in patients with morbid obesity is a chapter still to be written, as even elastosonographic methods have their limits. Although special XL probes, which seem to be able to reduce the need for nondiagnostic tests in subjects with severe obesity, have recently been used for morbidly obese patients, the values obtained are lower than those in nonobese subjects, and therefore, there are no well-defined cut-offs that can indicate the reliability of the method [15].

\section{Data Availability}

The data used to support the findings of this study are available from the corresponding author upon request.

\section{Conflicts of Interest}

The authors declare that they have no conflicts of interest.

\section{Acknowledgments}

This work was supported in part by the Fondazione Associazione Italiana per la Ricerca sul Cancro (AIRC; project no. 18394 , to M.C.).

\section{References}

[1] European Association for the Study of the Liver (EASL), European Association for the Study of Diabetes (EASD); European Association for the Study of Obesity (EASO), "EASL-EASDEASO Clinical Practice Guidelines for the management of non- alcoholic fatty liver disease," Journal of Hepatology, vol. 64, no. 6, pp. 1388-1402, 2016.

[2] National Institute for Health and Care Excellence (UK), "Nonalcoholic fatty liver disease: assessment and management," https://www.nice.org.uk/guidance/ng49.
[3] V. W. Wong, W. K. Chan, S. Chitturi et al., "Asia-Pacific working party on non-alcoholic fatty liver disease guidelines 2017part 1: definition, risk factors and assessment," Journal of Gastroenterology and Hepatology, vol. 33, no. 1, pp. 70-85, 2018.

[4] S. Chitturi, V. W. Wong, W. K. Chan et al., "The Asia-Pacific working party on non-alcoholic fatty liver disease guidelines 2017-part 2: management and special groups," Journal of Gastroenterology and Hepatology, vol. 33, no. 1, pp. 86-98, 2018.

[5] Italian Association for the Study of the Liver (AISF), "AISF position paper on nonalcoholic fatty liver disease (NAFLD): updates and future directions," Digestive and Liver Disease, vol. 49, pp. 471-483, 2017.

[6] N. Chalasani, Z. Younossi, J. E. Lavine et al., "The diagnosis and management of nonalcoholic fatty liver disease: practice guidance from the American Association for the Study of Liver Diseases," Hepatology, vol. 67, no. 1, pp. 328-357, 2018.

[7] M. Soresi, D. Noto, A. B. Cefalù et al., "Nonalcoholic fatty liver and metabolic syndrome in Italy: results from a multicentric study of the Italian Arteriosclerosis Society," Acta Diabetologica, vol. 50, no. 2, pp. 241-249, 2013.

[8] Z. Younossi, F. Tacke, M. Arrese et al., "Global perspectives on nonalcoholic fatty liver disease and nonalcoholic steatohepatitis," Hepatology, vol. 69, no. 6, pp. 2672-2682, 2019.

[9] M. C. S. Wong, J. L. W. Huang, J. George et al., "The changing epidemiology of liver diseases in the Asia-Pacific region," Nature Reviews Gastroenterology \& Hepatology, vol. 16, no. 1, pp. 57-73, 2019.

[10] J. Wu, J. You, L. Yerian, A. Shiba, P. R. Schauer, and D. I. Sessler, "Prevalence of liver steatosis and fibrosis and the diagnostic accuracy of ultrasound in bariatric surgery patients," Obesity Surgery, vol. 22, no. 2, pp. 240-247, 2012.

[11] A. A. de Moura, H. P. Cotrim, D. B. Barbosa et al., "Fatty liver disease in severe obese patients: diagnostic value of abdominal ultrasound," World Journal of Gastroenterology, vol. 14, no. 9, pp. 1415-1418, 2008.

[12] A. Petrick, P. Benotti, G. C. Wood et al., "Utility of ultrasound, transaminases, and visual inspection to assess nonalcoholic fatty liver disease in bariatric surgery patients," Obesity Surgery, vol. 25, no. 12, pp. 2368-2375, 2015.

[13] M. Soresi, L. Giannitrapani, A. M. Florena et al., "Reliability of the bright liver echo pattern in diagnosing steatosis in patients with cryptogenic and HCV-related hypertransaminasaemia," Clinical Radiology, vol. 64, no. 12, pp. 1181-1187, 2009.

[14] R. Hernaez, M. Lazo, S. Bonekamp et al., "Diagnostic accuracy and reliability of ultrasonography for the detection of fatty liver: a meta-analysis," Hepatology, vol. 54, no. 3, pp. 10821090, 2011.

[15] C. F. Dietrich, J. Bamber, A. Berzigotti et al., "EFSUMB guidelines and recommendations on the clinical use of liver ultrasound elastography, update 2017 (long version)," Ultraschall in der Medizin - European Journal of Ultrasound, vol. 38, no. 4, pp. e16-e47, 2017.

[16] R. K. Sterling, E. Lissen, N. Clumeck et al., "Development of a simple noninvasive index to predict significant fibrosis in patients with HIV/HCV coinfection," Hepatology, vol. 43, no. 6, pp. 1317-1325, 2006.

[17] S. A. Harrison, D. Oliver, H. L. Arnold, S. Gogia, and B. A. Neuschwander-Tetri, "Development and validation of a simple NALFD clinical scoring system for identifying patient without advanced disease," Gut, vol. 57, no. 10, pp. 1441$1447,2008$. 
[18] P. Angulo, J. M. Hui, G. Marchesini et al., "The NAFLD fibrosis score: a noninvasive system that identifies liver fibrosis in patients with NAFLD," Hepatology, vol. 45, no. 4, pp. 846854, 2007.

[19] G. Mancia, R. Fagard, K. Narkiewicz et al., "2013 ESH/ESC Practice Guidelines for the management of arterial hypertension," Blood Pressure, vol. 23, no. 1, pp. 3-16, 2014.

[20] J. J. Chamberlain, A. S. Rhinehart, C. F. Shaefer Jr., and A. Neuman, "Diagnosis and management of diabetes: synopsis of the 2016 American Diabetes Association Standards of Medical Care in Diabetes," Annals of Internal Medicine, vol. 164, no. 8, pp. 542-552, 2016.

[21] D. R. Matthews, J. P. Hosker, A. S. Rudenski, B. A. Naylor, D. F. Treacher, and R. C. Turner, "Homeostasis model assessment: insulin resistance and beta-cell function from fasting plasma glucose and insulin concentrations in man," Diabetologia, vol. 28, no. 7, pp. 412-419, 1985.

[22] D. E. Kleiner, E. M. Brunt, M. Van Natta et al., "Design and validation of a histological scoring system for nonalcoholic fatty liver disease," Hepatology, vol. 41, no. 6, pp. 1313-1321, 2005.

[23] P. Bedossa, "Utility and appropriateness of the fatty liver inhibition of progression (FLIP) algorithm and steatosis, activity, and fibrosis (SAF) score in the evaluation of biopsies of nonalcoholic fatty liver disease," Hepatology, vol. 60, no. 2, pp. 565575, 2014.

[24] M. H. Zweig and G. Campbell, "Receiver-operating characteristic (ROC) plots: a fundamental evaluation tool in clinical medicine," Clinical Chemistry, vol. 39, no. 4, pp. 561-577, 1993.

[25] R. Pezzilli, A. M. Morselli-Labate, R. Miniero, B. Barakat, M. Fiocchi, and O. Cappelletti, "Simultaneous serum assay of lipase and inteleukin-6 for early diagnosis of prognosis acute pancreatitis," Clinical Chemistry, vol. 45, no. 10, pp. 1762$1767,1999$.

[26] R. J. Liang, H. H. Wang, W. J. Lee, P. L. Liew, J. T. Lin, and M. S. Wu, "Diagnostic value of ultrasonographic examination for nonalcoholic steatohepatitis in morbidly obese patients undergoing laparoscopic bariatric surgery," Obesity Surgery, vol. 17, no. 1, pp. 45-56, 2007.

[27] C. C. Mottin, M. Moretto, A. V. Padoin et al., "The role of ultrasound in the diagnosis of hepatic steatosis in morbidly obese patients," Obesity Surgery, vol. 14, no. 5, pp. 635-637, 2004.

[28] J. J. Deeks and D. G. Altman, "Diagnostic tests 4: likelihood ratios," BMJ, vol. 329, no. 7458, pp. 168-169, 2004.

[29] T. A. Furukawa, S. Strauss, H. C. Bucher, and G. Guyatt, "Diagnostic test," in User's guides to the medical literature, G. Guyatt, D. Rennie, M. O. Meade, and D. J. Cook, Eds., pp. 223-250, McGraw-Hill Education, New York, 2014.

[30] V. Ratziu, P. Giral, F. Charlotte et al., "Liver fibrosis in overweight patients," Gastroenterology, vol. 118, no. 6, pp. 1117$1123,2000$.

[31] J. M. Clark, "The epidemiology of nonalcoholic fatty liver disease in adults," Journal of Clinical Gastroenterology, vol. 40, pp. 5-10, 2006.

[32] P. M. Gholam, L. Flancbaum, J. T. Machan, D. A. Charney, and D. P. Kotler, "Nonalcoholic fatty liver disease in severely obese subjects," The American Journal of Gastroenterology, vol. 102, no. 2, pp. 399-408, 2007.
[33] A. Losekann, A. C. Weston, A. A. de Mattos et al., "Non-alcoholic steatohepatitis (NASH): risk factors in morbidly obese patients," International Journal of Molecular Sciences, vol. 16, no. 10, pp. 25552-25559, 2015.

[34] M. Luger, R. Kruschitz, C. Kienbacher et al., "Prevalence of Liver Fibrosis and its Association with Non-invasive Fibrosis and Metabolic Markers in Morbidly Obese Patients with Vitamin D Deficiency," Obesity Surgery, vol. 26, no. 10, pp. 2425-2432, 2016.

[35] E. Bugianesi, A. J. McCullough, and G. Marchesini, "Insulin resistance: a metabolic pathway to chronic liver disease," Hepatology, vol. 42, no. 5, pp. 987-1000, 2005.

[36] E. Cazzo, J. C. Pareja, and E. A. Chaim, "Nonalcoholic fatty liver disease and bariatric surgery: a comprehensive review," São Paulo Medical Journal, vol. 135, no. 3, pp. 277-295, 2017.

[37] C. K. Argo, P. G. Northup, A. M. S. Al-Osaimi, and S. H. Caldwell, "Systematic review of risk factors for fibrosis progression in non-alcoholic steatohepatitis," Journal of Hepatology, vol. 51, no. 2, pp. 371-379, 2009.

[38] S. McPherson, T. Hardy, E. Henderson, A. D. Burt, C. P. Day, and Q. M. Anstee, "Evidence of NAFLD progression from steatosis to fibrosing-steatohepatitis using paired biopsies: implications for prognosis and clinical management," Journal of Hepatology, vol. 62, no. 5, pp. 1148-1155, 2015.

[39] S. Ballestri, F. Nascimbeni, and E. Baldelli, "NAFLD as a sexual dimorphic disease: role of gender and reproductive status in the development and progression of nonalcoholic fatty liver disease and inherent cardiovascular risk," Advances in Therapy, vol. 34, no. 6, pp. 1291-1326, 2017.

[40] L. Carulli, A. Lonardo, S. Lombardini, G. Marchesini, and P. Loria, "Gender, fatty liver and GGT," Hepatology, vol. 44, no. 1, pp. 278-279, 2006.

[41] J. D. Yang, M. F. Abdelmalek, H. Pang et al., "Gender and menopause impact severity of fibrosis among patients with nonalcoholic steatohepatitis," Hepatology, vol. 59, no. 4, pp. 1406-1414, 2014.

[42] Z. Wang, M. Xu, Z. Hu, and U. K. Shrestha, "Prevalence of nonalcoholic fatty liver disease and its metabolic risk factors in women of different ages and body mass index," Menopause, vol. 22, no. 6, pp. 667-673, 2015.

[43] B. J. Turner, C. P. Wang, T. V. Melhado, R. Bobadilla, M. K. Jain, and A. G. Singal, "Significant increase in risk of fibrosis or cirrhosis at time of HCV diagnosis for Hispanics with diabetes and obesity compared with other ethnic groups," Clinical Gastroenterology and Hepatology, vol. 17, no. 7, pp. 1356$1363,2019$. 